\title{
1.
}

\section{Entwicklung eines symmetrischen Ausdrucks für den Grad einer durch Elimination her- vorgehenden Gleichung.}

(Von Herrn Ferd. Minding, Professor an der Universität zu Dorpat.)

(Gelesen in der Sitzung der Petersburger Akademie der Wissenschaften am $\frac{\text { 24. Nov. }}{6 \text {. Decbr. }}$ 1843, und aus dem Bulletin übersetzt.)

Im 22. Bande des gegenwärtigen Journals habe ich ein neues Verfahren angegeben zur Bestimmung des Grades der Gleichung in $x$, welche durch Elimination von $y$ zwischen zwei algebraischen Gleichungen $f(x, y)=f=A_{0} y^{m}$ $+\boldsymbol{A}_{1} y^{m-1}+\ldots \boldsymbol{A}_{m}=\mathbf{0}$, und $\varphi(x, y)=\varphi=\boldsymbol{B}_{0} y^{n}+\boldsymbol{B}_{1} y^{n-1}+\ldots+\boldsymbol{B}_{n}=\mathbf{0}$ erhalten wird, wo die Buchstaben $\boldsymbol{A}$ und $\boldsymbol{B}$, mit angehängten Zeigern, beliebige ganze Polynome in $x$ bedeuten. Der folgende Aufsatz betrifft einige weitere Entwickelungen dieses Gegenstandes. Obgleich nämlich jenes Verfahren für die Leichtigkeit der Rechnung wohl nichts zu wünschen übrig lässt, so führt es doch nicht auf einen Ausdruck, welcher aus den durch beide Gleichungen gelieferten Elementen symmetrisch zusammengesetzt wäre. Nach der Natur der Sache muss es aber einen solchen Ausdruck geben, und es scheint nicht unerheblich, denselben zu entwickeln.

Werden, wie in dem genannten Artikel, die Wurzeln $y$ der Gleichung $p=\mathbf{0}$ durch $y_{1}, y_{2}, \ldots y_{n}$, die Wurzeln $y$ oder $\eta$ der Gleichung $f=\mathbf{0}$ durch $\eta_{1}, \eta_{2}, \ldots \eta^{m}$ bezeichnet, und ist $\psi x=0$ die Endgleichung, so hat man: $\psi x=A_{0}^{n} B_{0}^{m}\left[\left(y_{1}-\eta_{1}\right)\left(\gamma_{1}-\eta_{2}\right) \ldots\left(y_{1}-\eta_{m}\right)\right]\left[\left(y_{2}-\eta_{1}\right)\left(y_{2}-\eta_{2}\right) \ldots\left(y_{2}-\eta^{m}\right)\right]$ ..... $\left[\left(y_{n}-\eta_{1}\right)\left(y_{n}-\eta_{2}\right) \ldots .\left(y_{n}-\eta_{m}\right)\right]$

Man theile die Wurzeln $y$ in Gruppen nach ihren verschiedenen Graden: nämlich die Gleichung $\varphi=0$ habe $r_{1}$ Wurzeln $y$ vom Grade $h_{1}, r_{2}$ Wurzeln vom Grade $h_{2}, \ldots$ endlich $r_{i}$ Wurzeln vom Grade $h_{i}$, und es sei $h_{1}>h_{2} \ldots>h_{i}$; wo das Zeichen $>$ die Gleichheit ausschliesst. Auf gleiche Weise werden Crelle's Journal f. d. M. Bd. XXXI. Heft 1. 
sich die Wurzeln $\eta$ in eine Anzahl $(j)$ von Gruppen theilen, nämlich in $p_{1}$ Wurzeln vom Grade $\varepsilon_{1}, p_{2}$ vom Grade $\varepsilon_{2}, \ldots$ endlich $p_{j}$ vom Grade $\varepsilon_{j}$; es sei wiederum $\varepsilon_{1}>\varepsilon_{2} \ldots>\varepsilon_{j}$. Betrachtet man nun mit einiger Aufmerksamkeit den vorstehenden Ausdruck von $\psi x$, so sieht man, dass der Grad von $\psi x$ dargestellt wird durch eine Summe der Grade der Wurzeln $y$ und $\eta$, in welcher jede Wurzel aus einer der beiden Reihen $y$ und $\eta$ so viele Male vorkommt, als sich in der andern Reihe Wurzeln finden, deren Grad niedriger ist als jener der anfänglich gewählten Wurzel. Hieraus folgt der Lehrsatz:

Ordnet man die Wurzeln y und $\eta$ der vorgelegten Gleichungen in eine Reihe nach der absteigenden Folge ihrer Grade, so erhält man den Grad der Endgleichung $\psi \mathrm{x}=0$, indem man den Grad jeder Wurzel, sowohl aus der Reihe y, als aus $\eta$, mit der Anzahl der Wurzeln der andern Reihe, welche ihr in der vorausgesetzten Anordnung nachfolgen, multiplicirt, diese sämmtlichen Producte addirt und zu der Summe noch $\mathrm{na}_{0}+\mathrm{mb}_{0}$ hinzufügt; wo $\mathrm{a}_{0}$ und $\mathrm{b}_{0}$ die Grade con $\mathrm{A}_{0}$ und $\mathrm{B}_{0}$ andeuten.

Hierbei ist noch zu bemerken, dass es, wenn der Grad einiger $y$ dem Grade einiger $\eta$ gleich ist, für die Anwendung der vorstehenden Regel gleichgültig ist, ob man die $y$ den $\eta$ gleichen Grades vorangehen oder folgen lässt. Der Klarheit wegen sollen im Folgenden immer die $y$ den $\eta$ von gleichem Grade, oder die $h$ den ihnen gleichen $\varepsilon$ vorangestellt werden.

Sind also zwei Gleichungen vorgelegt, welche z. B. $i=3, j=4$ und $h_{1} \equiv \varepsilon_{1}>\varepsilon_{2}>h_{2}>h_{3} \bar{\equiv} \varepsilon_{3}>\varepsilon_{4}$ geben, so erhält man, da es $r_{1}, r_{2}, r_{3}$ Wurzeln $y$ beziehungsweise von den Graden $h_{1}, h_{2}, h_{3}$, und $p_{1}, p_{2}, p_{3}, p_{4}$ Wurzeln $\eta$ beziehungsweise von den Graden $\varepsilon_{1}, \varepsilon_{2}, \varepsilon_{3}, \varepsilon_{4}$ giebt, den Grad der Endgleichung ausgedrückt durch

$$
g=n a_{0}+m b_{0}+r_{1} h_{1} m+\left(r_{2} h_{2}+r_{3} h_{3}\right)\left(p_{3}+p_{4}\right)+\left(p_{1} \varepsilon_{1}+p_{2} \varepsilon_{2}\right)\left(r_{2}+r_{3}\right),
$$

wo $g$ allgemein diesen Grad bezeichnet und für $p_{1}+p_{2}+p_{3}+p_{4}$ sein Werth $m$, d. i. die Anzahl der Wurzeln $\eta$ gesetzt ist.

Um ein Beispiel in Zahlen zu geben, sei

$$
\begin{aligned}
& f=\overline{\left(x^{6}\right) y^{8}}+\left(x^{4}\right) y^{7}+\overline{\left(x^{9}\right) y^{6}}+\left(x^{8}\right) y^{5}+\overline{\left(x^{10}\right) \gamma^{4}}+\left(x^{8}\right) y^{3}+\left(x^{7}\right) y^{2}+\left(x^{5}\right) y+\overline{\left(x^{6}\right)}=0 \\
& \varphi=\overline{\left(x^{3}\right) y^{7}}+\overline{\left(x^{8}\right) y^{6}}+\left(x^{4}\right) y^{5}+\overline{\left(x^{7}\right) y^{4}}+\left(x^{6}\right) y^{3}+\left(x^{5}\right) y^{2}+\overline{\left(x^{4}\right) y}+\overline{\left(x^{2}\right)}=\mathbf{0} \text {. }
\end{aligned}
$$

In diesem Beispiel sind durch einen obern Querstrich die Glieder der Polynome $f$ und $\varphi$ bezeichnet, aus welchen sich die Grade der Wurzeln $\eta$ und $y$ ergeben, und welche ich deshalb gradbestimmende Glieder nennen 
will; die Unterscheidung derselben, übrigens sehr leicht, ist bei verschiedenen Untersuchungen wesentlich, wie man Seite 263 des 20. Bandes dieses Journals sehen kann, wo diese Glieder termini principales genannt sind. Es ergeben sich nämlich die Grade der Wurzeln $\eta$ und $y$ aus folgenden nach und nach sich darbietenden Gleichungen:

$$
\begin{gathered}
8 \varepsilon_{1}+6=6 \varepsilon_{1}+9, \quad 6 \varepsilon_{2}+9=4 \varepsilon_{2}+10, \quad 4 \varepsilon_{3}+10=6 ; \quad 7 h_{1}+3=6 h_{1}+8, \\
6 h_{2}+8=4 h_{2}+7, \quad 4 h_{3}+7=h_{3}+4, \quad h_{4}+4=2,
\end{gathered}
$$

woraus folgt:

$$
\begin{gathered}
\varepsilon_{1}=\frac{3}{2}, p_{1}=2 ; \quad \varepsilon_{2}=\frac{1}{2}, p_{2}=2 ; \varepsilon_{3}=-1, p_{3}=4 ; h_{1}=5, r_{1}=1 ; \\
h_{2}=-\frac{1}{2}, r_{2}=2 ; \quad h_{3}=-1, r_{3}=3 ; h_{4}=-2, r_{4}=1 ;
\end{gathered}
$$

mithin $h_{1}>\varepsilon_{1}>\varepsilon_{2}>h_{2}>h_{3}=\varepsilon_{3}>h_{4}$. Hieraus findet man den Grad $g$ der Endgleichung $\psi x=0$ :

$$
\begin{gathered}
g=7.6+8.3+8 h_{1}+2.4 h_{2}+2.6 \varepsilon_{1}+3.4 h_{3}+2.6 \varepsilon_{2}+4 \varepsilon_{3} \\
=66+40-4-12+18+6-4=110 .
\end{gathered}
$$

Im 6. Artikel des 22. Bandes dieses Journals ist für $g$ der Ausdruck gegeben worden: $g=m b_{0}+k_{1}+k_{2}+\ldots+k_{n}$, wo $k$ allgemein den Grad bezeichnet, welchen $f(x, y)$ erhält, wenn man darin für $y$ eine der Wurzeln $y$ vom Grade $h$ der Gleichung $\varphi(x, y)=0$ setzt. Dieser Aufsatz bedarf einer passenden Umgestaltung, um mit dem vorstehend Entwickelten verglichen $\mathrm{zu}$ werden.

$$
\begin{aligned}
& \text { Man schreibe }\left(x^{a_{0}}\right)\left(x^{a_{1}}\right) \ldots\left(x^{a_{m}}\right)\left(x^{b_{0}}\right)\left(x^{b_{1}}\right) \ldots\left(x^{b_{n}}\right) \\
& \begin{array}{lllllllll}
\text { anstatt } & A_{0} & A_{1} & \ldots & A_{m} & B_{0} & B_{1} & \ldots & B_{n}
\end{array}
\end{aligned}
$$

so dass $a_{0}, a_{1}, \ldots b_{n}$ die Grade der Polynome $A_{0}, A_{1}, \ldots B_{n}$ ausdrücken. Es

$$
\text { seien }\left(x^{b}\right) y^{n},\left(x^{b} \lambda_{1}\right) y^{n-\lambda_{1}},\left(x^{b} \lambda_{2}\right) y^{n-\lambda_{2}} \ldots \ldots\left(x^{b} \lambda_{i-1}\right) y^{n-\lambda_{i-1}},\left(x^{b}\right)
$$

die gradbestimmenden Glieder von $\varphi\left(x_{1} y\right)$, nach der Reihe gestellt, nämlich so, dass

$$
0<\lambda_{1}<\lambda_{2} \ldots<\lambda_{i-1}<n \text { ist. }
$$

Man sieht nämlich leicht, dass das erste und letzte Glied des Polynoms $\varphi(x, y)$ immer zu diesen gradbestimmenden Gliedern gehören, und ich füge noch die leicht zu beweisende Bemerkung hinzu, dass wenn $b_{\lambda^{\prime}}$ die grösste unter den Zahlen $b_{0}, b_{1}, b_{2}, b_{3}, \ldots . b_{n}$ ist, das Glied $\left(x^{b} \lambda^{\prime}\right) y^{m-\lambda^{\prime}}$ von $\varphi$ ebenfalls ein gradbestimmendes sein wird. Wenn es in der Reihe $b_{0}, b_{1}, \ldots b_{n}$ mehrere Zahlen $b_{\lambda^{\prime}}, b_{\lambda^{\prime \prime}}, \ldots b_{\lambda^{u}}$ giebt, die einander gleich sind, und grösser als alle übrigen Zahlen dieser Reihe, so sind unter der Voraussetzung, dass $\lambda^{\prime}<\lambda^{\prime \prime} \ldots<\lambda^{u}$ sei, das erste und das letzte der Glieder $\left(x^{b} \lambda^{\prime}\right) y^{n-\lambda^{\prime}},\left(x^{b} \lambda^{\prime \prime}\right) y^{n-\lambda^{\prime \prime}}, \ldots \ldots\left(x^{b} \lambda^{u}\right) y^{n-\lambda_{u}}$ gradbestimmende, die andern aber sind es nicht. 
Allgemein liefert jedes Glied der Reihe $n h+b_{0},\left(n-\lambda_{1}\right) h+b_{\lambda_{1}}, \ldots\left(n-\lambda_{\varsigma}\right) h+b_{\lambda_{\varsigma}}, \ldots\left(n-\lambda_{i-1}\right) h+b_{\lambda_{i-1}}, b_{n}$, mit Ausnahme des ersten : und des letzten, zwei verschiedene Werthe von $h$, je nachdem es dem ihm vorangehenden oder nachfolgenden gleichgesetzt wird. Denn es ist $\left(n-\lambda_{\varsigma-1}\right) h \rho+b_{\lambda_{s-1}}=\left(n-\lambda_{\varsigma}\right) h+b_{\lambda_{\varsigma}}$ und $\left(n-\lambda_{\varsigma}\right) h_{s+1}+b_{\lambda_{\varsigma}}$ $=\left(n-\lambda_{\varsigma+1}\right) h_{\varsigma+1}+b_{\lambda_{\varsigma+1}}$, und $h_{\varsigma}>h_{\varsigma+1}$. Liegt nun der Grad $\varepsilon$ einer Wurzel $\eta$ zwischen $h_{\S}$ und $h_{\varsigma+1}$, oder ist er $=h_{\varsigma}$, so sieht man leicht, dass unter den Ausdrücken

$$
n_{\varepsilon}+b_{0}, \quad(n-1)_{\varepsilon}+b_{1}, \quad(n-2) \varepsilon+b_{2}, \ldots \ldots+b_{n-1}, \quad b_{n},
$$

$\left(n-\lambda_{\varsigma}\right)_{\varepsilon}+b_{\lambda_{\varsigma}}$ den grössten Werth hat, oder wenigstens, wenn $\varepsilon=h_{\varsigma}$, dem Werthe von $\left(n-\lambda_{\varsigma-1}\right) \varepsilon+b_{\lambda_{\xi-1}}$ und vielleicht noch einigen andern Werthen von $(n-\lambda) \varepsilon+b_{\lambda}$, deren $\lambda$ zwischen $\lambda_{\varsigma-1}$ und $\lambda_{\varsigma}$ liegen, gleich ist; aber grösser als die übrigen Glieder obiger Reihe. Folglich ist, wenn $l$ den Grad bezeichnet, den $\varphi$ erhält, wenn darin für $y$ eine Wurzel $\eta$ vom Grade $\varepsilon$ gesetzt wird, $l=\left(n-\lambda_{\varsigma}\right) \varepsilon+b_{\lambda_{\varsigma}}$. Ist $\varepsilon=h_{\varsigma}$, so hat man auch noch $l=$ $\left(n-\lambda_{s-1}\right) \varepsilon+b_{\lambda_{\xi-1}}$, und da es für das Folgende nöthig ist, zwischen diesen Ausdrücken von $l \mathrm{zu}$ wählen, so sei festgesetzt, dass immer der erste gewählt werde, $d$. $h$. derjenige, welcher den grössten Werthen von $\rho$ und $\lambda_{\varsigma}$ entspricht. Bezeichnen nun $l_{1} l_{2} \ldots l_{j}$ die beziehungsweise zu $\varepsilon_{1} \varepsilon_{\mathbf{2}} \ldots \varepsilon_{j}$ gehörigen $l$, und bedenkt man, dass $p_{1}$. Wurzeln $\eta$ vom Grade $\varepsilon_{1}, \ldots$ vorhanden sind, so verwandelt sich der im genannten Artikel gegebene Ausdruck von $\boldsymbol{g}$ in folgenden:

$$
g=n a_{0}+p_{1} l_{1}+p_{2} l_{2}+\ldots+p_{i} l_{j}=n a_{0}+\sum_{\gamma=1}^{\gamma=j}\left(p_{\gamma} l_{\gamma}\right) \text {. }
$$

Setzt man für $l_{\gamma}$ seinen Werth $\left(n-\lambda_{\varsigma}\right) \varepsilon_{\gamma}+b_{\lambda_{\varsigma}}$, so folgt hieraus

$$
g=n a_{0}+\sum_{\gamma=1}^{\gamma=j}\left(p_{\gamma} b_{\lambda_{\varsigma}}\right)+\sum_{\gamma=1}^{\gamma=j}\left\{\left(n-\lambda_{\varsigma}\right) p_{\gamma} \varepsilon_{\gamma}\right\}
$$

wo immer $\rho$ durch die Bedingung $h_{\varsigma} \bar{\equiv} \varepsilon \gamma>h_{\varsigma+1}$ bestimmt ist.

Sind eben so $\left(x^{a_{0}}\right) y^{m},\left(x_{\theta_{1}}^{a_{1}}\right) y^{m-\theta_{1}}, \ldots\left(x^{a_{\theta_{v}}}\right) y^{m-\theta_{\nu}}, \ldots\left(x^{a_{j-1}}\right)$ $y^{m-\theta_{j-1}},\left(x^{a_{m}}\right)$ die gradbestimmenden Glieder von $f$, und ist $0<\theta_{1}<\theta_{2} \ldots$ $<\theta_{\varsigma-1}<m$; ist ferner $h$ der Grad einer Wurzel $y$ von $\varphi=0$, die entweder zwischen $\varepsilon_{\nu}$ und $\varepsilon_{\nu+1}$ liege, oder gleich $\varepsilon_{\nu+1}$ sei: so wird der Grad, den $f$ durch Einsetzung einer Wurzel $y$ vom Grade $h$ von $\varphi=0$, anstatt $y$, erhält, ausgedrückt durch $k=\left(m-\theta_{v}\right) h+a_{\theta_{\nu}}$. Ist $h=\varepsilon_{v+1}$, so hat man noch $k=\left(m-\theta_{v+1}\right) h+a_{\theta_{v+1}}$, weil $\left(m-\theta_{v}\right) \varepsilon_{\nu+1}+a_{\theta_{\nu}}=\left(m-\theta_{v+1}\right) \varepsilon_{\nu+1}$ 
$+a_{\theta_{\nu+1}}$. In diesem Falle soll die Wahl des Zeigers $\nu$ immer den kleinsten Werth desselben treffen, welcher den kleinsten Werth von $\theta_{\nu}$ mit sich führt. Der Ausdruck von $g$ wird daher, dem vorigen entsprechend, wenn man noch erwägt, dass es $r_{1}$ Wurzeln $y$ vom Grade $h_{1}$ giebt, welche für den Grad von $f$ den Werth $k_{1}$ geben, u. s. f., folgender:

$$
g=m b_{0}+\sum_{\gamma=1}^{\gamma=i}\left(v_{\gamma} k_{\gamma}\right) \text { oder } g=m b_{0}+\sum_{\gamma=1}^{\gamma=i}\left(r_{\gamma} a_{\theta_{\nu}}\right)+\sum_{\gamma=1}^{\gamma=i}\left\{\left(m-\theta_{\nu}\right) r_{\gamma} h_{\gamma}\right\} \quad I I .
$$

wo $v$ durch die Bedingung $\varepsilon_{\nu}>h \gamma \equiv \varepsilon_{\nu+1}$ bestimmt ist.

Gehen wir nun auf die im Anfange dieses Aufsatzes ausgesprochene Regel zurück. Wenn man die Werthe von $h$ und $\varepsilon$ nach ihrer Grösse fallend ordnet, und zu grösserer Klarheit jedes $h$ so oft schreibt, als es Wurzeln $y$ vom Grade $h$ giebt, also $r$ mal, und jedes $\varepsilon, p$ mal; wenn man ferner, im Falle der Gleichheit eines $h$ mit einem $\varepsilon$, jenes diesem voranstcllt; so braucht man nur zu zählen, wie viele $h$ jedem $\varepsilon$ und wie viele $\varepsilon$ jedem $h$ folgen. Man sieht aber leicht, dass die Anzahl der auf irgend ein $h$, nämlich auf $h_{\gamma}$, folgenden $\varepsilon, m-\theta_{\nu}$ ist, wo $\theta_{\nu}$ genau in dem Sinne zu nehmen ist, welcher ihm in dem Ausdrucke II. zukommt. Denn da $h_{\gamma}$ kleiner ist als $\varepsilon_{v}$ und grösser als $\varepsilon_{\nu+1}$, oder gleich $\varepsilon_{\nu+1}$, so sind die auf $h_{\gamma}$ folgenden $\varepsilon$ diese: $\varepsilon_{\nu+1} \varepsilon_{\nu+2} \ldots \varepsilon_{i}$; wovon jedes beziehungsweise $p_{\nu+1} p_{\nu+2} \ldots p_{j}$ mal zu schreiben ist. Man hat aber $\left(m-\theta_{v}\right) \varepsilon_{\nu+1}+a_{\theta_{\nu+1}}=\left(m-\theta_{\nu+1}\right) \varepsilon_{\nu+1}+a^{\theta}{ }_{\nu+1}$, $\ldots . .,\left(m-\theta_{j-1}\right) \varepsilon_{j}+a_{\theta_{j-1}}=a_{m}$, und $p_{v+1}=\theta_{\nu+1}-\theta_{v}, \quad p_{v+2}=a_{\theta_{v+2}}$ $a_{\theta_{\nu+1}}, \ldots \ldots, p_{i}=m-\theta_{j-1}$, folglich $p_{\nu+1}+p_{\nu+2}+\ldots+p_{j}=\mathrm{m}-\theta v$, w. z. B. $\mathbf{n}$.

Ebenso ist die Anzahl der auf $\varepsilon_{\nu}$ folgenden $h$ gleich $n-\lambda_{\varsigma}, \lambda_{\varsigma}$ in dem Sinne des Ausdrucks $I$. genommen; denn da $\varepsilon_{\nu} \equiv h_{\varsigma}$ und $\varepsilon_{\nu}>h_{\varsigma+1}$, so sind $h_{\varsigma+1} h_{\varsigma+2} \ldots h_{i}$ die auf $\varepsilon_{v}$ folgenden $h$; ihre Anzahl ist $r_{\varsigma+1}+r_{\varsigma+2}+\ldots$ $+r_{i}=n-\lambda_{5}$.

Fasst man das Vorstehende zusammen, so gelangt man zu einem neuen Ausdruck von $g$, nämlich :

$$
g=n a_{0}+m b_{0}+\sum_{\gamma=1}^{\gamma=i}\left\{\left(m-\theta_{\nu}\right) r_{\gamma} h_{\gamma}\right\}+\sum_{\gamma=1}^{\gamma=j}\left\{\left(n-\lambda_{\varsigma}\right) p_{\gamma} \varepsilon_{\gamma}\right\} \quad \text { III. }
$$

wo die durch $\Sigma$ bezeichneten Summen genau die nämlichen sind wie in $I$. und II. Daher folgt durch Vergleichung von $I$. und II. mit III.

$$
\left.\left.\sum_{\gamma=1}^{\gamma=i}\left(r_{\gamma} a_{\theta_{\nu}}\right)=n a_{0}+\sum_{\gamma=1}^{\gamma=j}\left\{n-\lambda_{\varsigma}\right) p_{\gamma} s_{\gamma}\right\}, \sum_{\gamma=1}^{\gamma=j}\left(p_{\gamma} b_{\lambda_{\zeta}}\right)=m b_{0}+\sum_{\gamma=1}^{\gamma=i}\left\{\left(m-\theta_{\nu}\right) r_{\gamma} h_{\gamma}\right)\right\}
$$


und schliesslich ein neuer Ausdruck von g, nämlich:

$$
g=\sum_{\gamma=1}^{\gamma=\mathbf{i}}\left(r_{\gamma} a_{\theta_{\nu}}\right)+\sum_{\gamma=1}^{\gamma=j}\left(p_{\gamma} b_{\lambda_{s}}\right) . \quad \quad I V .
$$

Zufolge dieser bemerkenswerthen Formel ist der Grad der Endgleichung in $x$ die Summe der Grade einiger der nach $x$ geordneten Coëfficienten der beiden Gleichungen, nämlich der zu denjenigen Gliedern gehörigen, aus welchen sich für jede Wurzel $\eta$ oder. $y$ die Grade der Functionen $\varphi(x, \eta)$ und $f(x, y)$ ergeben, wo $\eta$ Wurzeln von $f(x, y)=0$, y Wurzeln von $\varphi(x, y)=0$ sind. Wenn diese Grade von $\varphi(x, y)$ und $f^{\prime}(x, y)$ sich aus mehrern Gliedern herleiten lassen, so wird alle Ungewissheit vermieden, wenn man in dem einen der Polynome $f$ und $\varphi$ unter den verschiedenen zulässlichen Glie'dern das am weitesten rechterhand, und zugleich im andern das am weitesten linkerhand stehende auswählt.

In dem obigen Zahlenbeispiele ist

$$
\begin{gathered}
\sum_{\gamma=1}^{\gamma=i}\left(r_{\gamma} a_{\theta_{\nu}}\right)=a_{0}+2 a_{4}+3 a_{4}+a_{8}=6+2.10+3.10+6=62 \\
\sum_{\gamma=1}^{\gamma=j}\left(p_{\gamma} b \lambda_{\varsigma}\right)=2 b_{1}+2 b_{1}+4 b_{6}=2.8+2.8+4.4=48 \\
\text { daher } g=62+48=110 .
\end{gathered}
$$

In dem mehrerwähnten Artikel wurde bemerkt, dass nach Elimination von $x$ zwischen $f=0$ und $\varphi=0$, der Grad $g^{\prime}$ der Endgleichung in $y$ dem Grade $g$ der Endgleichung in $x$ immer gleich ist, wenn $\left(x^{a}\right)$ und $\left(x^{b}\right)$ keinen gemeinschaftlichen Factor haben, und wenn dieselbe Voraussetzung auch von den Coëfficienten der höchsten Potenzen von $x$ in beiden nach $x$ geordneten Gleichungen gilt. Dies folgt ganz einfach daraus, dass unter den angegebenen Bedingungen das vorgelegte System keine andere als endliche Lösungen hat. Man kann jedoch einen, die nähern Umstände darlegenden Beweis dieses Satzes wünschen.

Um das Polynom $\varphi=\left(x^{b}\right) y^{n}+\left(x^{b}\right) y^{n-1}+\ldots+\left(x^{b_{n}}\right)$ nach $x$ zu ordnen, gehe man in der Reihe der Zahlen $b_{0}, b_{1}, b_{2}, \ldots$ bis zu derjenigen $b_{t}$ fort, welche grösser ist als alle ihr links vorangehenden, und nicht kleiner als 
irgend eine der ihr folgenden, nämlich $b_{t_{1}+1}, b_{t+2}, \ldots b_{n}$. Alsdann suche man in der Reihe $b_{0}, b_{1}, b_{2}, \ldots b_{t-1}$ diejenige Zahl $b_{t_{1}}$ welche grösser ist als alle ihr links vorangehenden, und nicht kleiner als irgend eine der ihr rechts nachfolgenden $b_{t_{1}+1} \ldots b_{t-1}$; in der Reihe $b_{0}, b_{1}, \ldots b_{t_{1}-1}$ suche man hierauf die Zahl $b_{t_{2}}$, welche grösser als alle ihr vorangehenden und nicht kleiner als eine der ihr folgenden ist, und so fort, bis man auf $b_{0}$ kommt, welche die letzte der Zahlen $b_{t}, b_{t_{1}}, b_{t_{2}}, \ldots b_{0}$ sein wird. Das Polynom $\varphi$, nach $x$ geordnet, muss nun nothwendig folgende Glieder enthalten:

$$
\left(y^{n-t}\right) x^{b_{t}}\left(y^{n-t_{1}}\right) x^{b_{t_{1}}}\left(y^{n-t_{2}}\right) x^{b_{t_{2}}} \ldots \ldots\left(y^{n}\right) x^{b_{0}}
$$

wo $\left(y^{\mu}\right)$, wie immer, ein Polynom vom Grade $\mu$ andeutet. Wird noch durch das nämliche Zeichen, mit einem Puncte versehen, nämlich durch $\left(\dot{y}^{\mu}\right)$ ein Polynom bezeichnet, dessen Grad gleich $\mu$ oder niedriger als $\mu$ ist, so nimmt das Polynom $\varphi$, nach $x$ geordnet, folgende allgemeine Gestalt an:

$$
\begin{gathered}
\varphi=\left(y^{n-t}\right) x^{b_{t}}+\left(\dot{y}^{n-t}\right) x^{b_{t}-1}+\left(\dot{y}^{n-t}\right) x^{b_{t}-2}+\ldots+\left(\dot{y}^{n-t}\right) x^{b_{t}+1} \\
+\left(y^{n-t_{1}}\right) x^{b_{1}}+\left(\dot{y}^{n-t_{1}}\right) x^{b_{1}-1}+\ldots+\left(y^{n-t_{2}}\right) x^{b_{t}}+\left(\dot{y}^{n-t_{2}}\right) x^{b_{t_{2}-1}}+\ldots+\left(y^{n}\right) x^{b_{0}} \\
+\left(\dot{y}^{n}\right) x^{b_{0}-1}+\left(\dot{y}^{n}\right) x^{b_{0}-2}+\ldots+\left(\dot{y}^{n}\right)
\end{gathered}
$$

wo die zwischen die Glieder der Reihe (a) eingeschalteten Glieder sämmtlich mit Puncten versehen sind, weil die Grade ihrer Coëfficienten den in den Klammern befindlichen zugehörigen Exponenten höchstens gleich sein können, aber nicht nothwendig gleich sind.

Es ist nun klar, dass keines der vor $\left(y^{n}\right) x^{b_{0}}$ eingeschalteten Glieder ein gradbestirnmendes Glied der nach $x$ geordneten Gleichung $\varphi=0$ sein kann, und dass die gradbestimmenden Glieder dieser Gleichung alle entweder in der Reihe $(a)$ enthalten sind, oder sich unter den in vorstehendem Ausdrucke von $\varphi$ auf $\left(y^{n}\right) x^{b}$ folgenden Gliedern befinden. Man erkennt noch sogleich, dass $\left(y^{n-t}\right) x^{b},\left(y^{n}\right) x^{b}$ und das letzte Glied $\left(\dot{y}^{n}\right)$ immer gradbestimmende Glieder von $\varphi$ sein müssen. Es soll nun gezeigt werden, dass, wenn $\left(y^{n-t_{v}}\right) x^{b_{t}}$ e eines der Glieder $(a)$ ist, mit Ausnahme des letzten $\left.\left(y^{n}\right) x^{b_{0}}\right)$ und zugleich ein gradbestimmendes Glied der nach $x$ geordneten Gleichung $\varphi=0$, dass alsdann $\left(x^{b_{t}}\right) \boldsymbol{r}^{n-t} v$ ein gradbestimmendes Glied derselben Gleichung nach $y$ geordnet ist, und umgekehrt.

Denn es sei $\left(y^{n-t^{\prime}}\right) x^{b_{t^{\prime}}}$ das gradbestimmende Glied, welches auf das vorhergenannte in der nach $x$ geordneten Gleichung $\varphi=0$ folgt, und es sei $s$ der Grad von $x$, welcher aus der Vergleichung dieser beiden Glieder hervorgeht, so hat man $b_{t_{v}} \cdot s+n-t_{v}=b_{t_{v}} \cdot s+n-t_{\dot{v}}=u$, und der Werth von 
$s$ ist positiv, weil $v^{\prime}>v$, mithin $t_{v}<t_{v}, b_{t^{\prime}}<b_{t_{v}}$ ist. Bezeichnet noch $\sigma$ die Anzahl der Wurzeln $x$ von $\varphi=0$, deren Grad $s$ ist, so ist $\sigma=b_{t v}-b_{t \dot{v}^{\prime}}$. Nach den Eigenschaften der gradbestimmenden Glieder ist aber $u \bar{\equiv} b_{t_{\omega}} \cdot s+n$ - $t_{\omega}$, wenn $\omega$ eine Zahl zwischen $v$ und $v^{\prime}$, also $t_{\omega}$ eine Zahl zwischen $t_{v}$ und $t_{i}$ ist; dagegen ist $u>b_{t_{\omega}} \cdot s+n-t_{\omega}$, wenn $\omega$ ausserhalb der Grenzen $v$ und $v^{\prime}$ liegt; $\omega$ bezeichnet hier eine der Zahlen $0,1,2 \ldots \delta$, und es ist $t_{0}=t, t_{\delta}=0 ; \delta+1$ ist die Anzahl der Glieder der Reihe (a). Dividirt man nun vorstehende Ausdrücke durch $s$ und setzt $\frac{1}{s}=h, \frac{u}{s}=h u=u ́$, so komint $\left(n-t_{v}\right) h+b_{t_{v}}=\left(n-t_{i}\right) h+b_{t_{v}}=\dot{u}, \dot{u} \bar{\equiv}\left(n-t_{\omega}\right) h+b_{t_{\omega}}$ wenn $\omega$ zwischen $v$ und $v^{\prime}, \dot{u}>\left(n-t_{\omega}\right) h+b_{t_{\omega}}$, wenn $\omega$ ausserhalb der Grenzen $v$ und $v^{\prime}$ liegt.

Ist $\varrho$ eine Zahl der Reihe 1, 2, 3, . n , welche in $t_{\delta}, t_{\delta-1} \ldots t_{2}, t_{1}, t$ nicht vorkommt, und liegt $\varrho$ zwischen $t_{\omega}$ und $t_{\omega+1}$, so dass $t_{\omega}>\rho>t_{\omega+1}$, so ist auch $b_{\varsigma}<b_{t_{\omega}}, b_{\varsigma}<b_{t_{\omega+1}}$, folglich, weil nach der Voraussetzung $u \bar{\Sigma}\left(n-t_{\omega+1}\right) h$ $+b_{t_{\omega}+1}$, so ist auch $u>(n-s) h+b_{\varsigma}$. Ist $\varrho$ grösser als $t_{0}$ oder $t$, so ist $b_{\varsigma} \equiv b_{t}$ und weil $u^{\prime} \bar{\Sigma}(n-t) h+b_{t}$, so ist $u^{\prime}>(\mathrm{n}-s) h+b_{\varsigma}$.

Hierdurch ist bewiesen, dass das Glied $\left(x^{b_{t}} v\right) y^{n-t} v$, welches in der nach $y$ geordneten Gleichuug $\varphi=0$ nothwendig vorkommt, auch ein gradbestimmendes Glied derselben ist, wenn $\left(y^{n-t} v\right) x^{b_{b}}{ }_{v}$ ein gradbestimmendes Glied der nach $x$ geordneten Gleichung $\varphi=0$ (aus der Reihe $[a]$ ) war. Da die Urnkehrung eben so richtig ist, so folgt: Wenn $\left(x^{b}\right) y^{n},\left(x^{b} \lambda_{1}\right) y^{n-\lambda_{1}},\left(x^{b} \lambda_{2}\right) y^{n-\lambda_{2}}$ ... $\left(x^{b_{t}}\right) y^{n-t}(b)$ die Reihe der gradbestimmenden Glieder des nach $y$ geordneten $\varphi$ ist, fortgesetzt bis zu dem Gliede $\left(x^{b_{t}}\right) y^{n-t}$, welches dadurch bestimmt ist, dass $b_{t}>b_{\varsigma}$ wenn $s<t_{1}$ und $b_{t}>b_{\varsigma}$ wenn $\varsigma>t$; so stellt $\left(y^{n-t}\right) x^{b_{t}} \ldots$, $\left(y^{n-\lambda_{2}}\right) x^{b} \lambda_{2},\left(y^{n-\lambda_{1}}\right) x^{b} \lambda_{1},\left(y^{n}\right) x^{b}(c)$ die Reihe der gradbestimmenden Glieder des nach $x$ geordneten $\varphi$ dar, jedoch nur bis zum Gliede $\left(y^{n}\right) x^{b}{ }_{0}$. Die Vergleichung der Glieder (b) liefert die positiven Grade der Wurzeln $y$ der Gleichung $\varphi=0$; die folgenden gradbestimmenden Glieder dieser Gleichung, welche in ( $b$ ) weggelassen sind, liefern die Grade von $y$, welche Null oder negativ sind.

Wenn nun $h_{1}, h_{2}, \ldots h_{\gamma}$ die in der Reihe $h_{1}, h_{2}, \ldots h_{i}$ enthaltenen positiven Grade vorstellen, während die übrigen Grade $h_{\gamma+1} h_{\gamma+2} \ldots h_{i}$ Null oder negativ sind, und wenn, wie früher, die Gleichung $q=0$, nach $y$ auf- 
gelöset, $r_{1}$ Wurzeln vom Grade $h_{\mathbf{1}}$ u. s. f. darbietet; so wird die nämliche Gleichung, nach $x$ aufgelöset, $r_{1} h_{1}$ Wurzeln vom Grade $\frac{1}{h_{1}}, r_{2} h_{2}$ vom Grade $\frac{1}{h_{2}}, \ldots r_{\gamma} h_{\gamma}$ Wurzeln vom Grade $\frac{1}{h_{y}}$ darbieten; ihre übrigen Wurzeln aber, wenn es deren giebt, sind vom Grade Null, oder von negativen Graden.

Denn man hatte vorhin, um den Grad $s$ von $x$ in $y$ zu bestimmen, $\left(b_{t_{v}}-b_{t_{v}^{\prime}}\right) s=t_{v}-t_{v}$ und $\sigma=b_{t_{v}}-b_{t_{v}}$, wo $\sigma$ die Anzahl der Wurzeln $x$ vom Grade $s$ ist. Eben so hat man zur Bestimmung von $h:\left(t_{v}-t_{i}\right) h=$ $b_{t_{v}}-b_{t_{v}}$ und $r=t_{v}-t_{i}=$ Anzahl der Wurzeln vom Grade $h$; folglich $\sigma=$ $r h$ und $s=\frac{1}{h}$. Hiermit ist der Haupttheil des Satzes bewiesen, und das Uebrige leuchtet ein, weil die Vergleichung der Glieder $\left(y^{n}\right) x^{b}$ und der folgenden bis $\left(\dot{y}^{n}\right)$ für die Grade nur negative Werthe oder Null geben kann.

Denken wir uns also das vorgelegte System $f=\left(x^{a_{0}}\right) y^{m}+\ldots .+\left(x^{a_{m}}\right)$ $=0, \varphi=\left(x^{b}\right) y^{n}+\ldots+\left(x^{b}\right)=0$ durch Anordnung nach $x$ übergehend in $f=\left(y^{\alpha_{0}}\right) x^{\mu}+\left(y^{\alpha_{1}}\right) x^{\mu-1}+\ldots+\left(y^{\alpha_{\mu}}\right)=0, \quad \varphi=\left(y^{\beta_{0}}\right) x^{\nu}+\left(y^{\beta_{1}}\right) x^{\nu-1}+\ldots$ $+\left(y^{\beta_{\nu}}\right)=0$. Es wird angenommen, dass $\left(x^{a}\right)$ und $\left(x^{b_{0}}\right)$ keinen Factor gemein haben; damit aber auch $\left(y^{\alpha_{0}}\right)$ und $\left(y^{\beta_{0}}\right)$ nicht beide durch $y$ theilbar seien, muss noch entweder $\mu=a_{m}$, oder $\nu=b_{n}$ sein; es sei also $\mu=a_{m}$, während $b_{n}$ gleich $\nu$ oder auch kleiner als $\nu$ ist; ferner muss noch $\alpha_{\mu}=m$, oder $\beta_{\nu}=n$ sein, denn fände keins von beiden Statt, so wären $\left(x^{a}{ }^{a}\right)$ und $\left(x^{b 0}\right)$ beide durch $x$ theilbar.

Da $\mu=a_{m}$, so hat die Gleichung $f=0$ keine Wurzeln $y$ von negativen Graden; von den Wurzeln $y^{\prime}$ von $\varphi=0$ seien $h_{1}, h_{2} \ldots h_{\gamma}$ die positiven Grade, die übrigen Grade $h_{\gamma+1}, h_{\gamma+2}, \ldots h_{i}$ sind der erste Null oder negativ, die folgenden alle negativ. Ist nun $h$ einer der Grade $h_{1}, h_{2} \ldots h_{\gamma}$, so ist $k=(m-t) h+a_{t}$ der Grad, den $f$ erhält, wenn darin für $y$ eine Wurzel $y$ von $\varphi=0$ vom Grade $x$ gesetzt wird; $t$ ist ein gehörig zu wählender Zeiger aus der Reihe $0, \theta_{1}, \theta_{2}, \ldots \theta_{j-1}, m$, welche den gradbestimmenden Gliedern von $f$ entspricht. Ist hingegen $h=0$ oder negativ, so ist offenbar $k=a_{m}=\mu$. Hieraus ergiebt sich für den Grad der Endgleichung in $x: g=m b_{0}+r_{1} k_{1}+\ldots$ $+r_{i} k_{i}$ oder $g=m b_{0}+r_{1}\left(\left(m-t_{1}\right) h+a_{t_{1}}\right)+r_{2}\left(\left(\mathrm{~m}-t_{2}\right) h+a_{t_{2}}\right) \ldots \ldots$ $+r_{\gamma}\left(\left(m-t_{\gamma}\right) h_{\gamma}+a_{t_{\gamma}}\right)+\left(r_{\gamma+1}+r_{\gamma+2}+. . r_{i}\right) a_{m}$. Die Anzahl der Wurzeln von nicht positiven Graden ist aber $\beta_{0}$, also $r_{\gamma+1}+. .+r_{i}=\beta_{0}$; folglich ist

$$
g=m b_{0}+\mu \beta_{0}+r_{1}\left(\left(m-t_{1}\right) h+a_{t_{1}}\right)+\ldots+r_{\gamma}\left(\left(m-t_{\gamma}\right) h_{\gamma}+a_{t_{\gamma}}\right)
$$

Crelle's Journal f. d. M. Bd. XXXI. Heft 1. 
Andrerseits theilen sich die Wurzeln $x$ von $\varphi=\mathbf{0}$, wie gezeigt ist, in $r_{1} h_{1}$ vom Grade $\frac{1}{h}, \ldots r_{\gamma} h_{\gamma}$ vom Grade $\frac{1}{h_{\gamma}}$; die Grade der übrigen Wurzeln, wenn es deren giebt, sind alle Null, wenn $a_{\mu}=m$, hingegen Null oder negativ, wenn $a_{\mu}<m$. Nun sieht man aber sofort, dass, wenn $(m-t) h+a_{t}$ den Grad von $f$ für einen Werth von $y$ in $x$ vom Grade $h$ ausdrückt, $a_{t} \cdot \frac{1}{h}+m-t$ der Grad von $f$ für einen Werth von $x$ in $y$ vom Grade $\frac{1}{h}$ sein wird, wo $t$ dieselbe Zahl ist, wie vorher; ferner wird für eine Wurzel $x$, deren Grad Null oder negativ ist, der Grad von $f$ in $y$ gleich $m$, wenn $\alpha_{\mu}$ $=m$; ist $\alpha_{\mu}<m$, so muss $\beta_{\nu}=n$ sein; alsdann hat $\varphi=0$ keine Wurzeln $x$ von negativen Graden, und die Wurzeln vom Grade Null geben immer den Grad von $f$ gleich $m$. Bemerkt man noch, dass die Anzahl der Wurzeln $x$ von $\varphi=0$., deren Grad Null oder negativ, gleich $b_{0}$ ist, so ergiebt sich für den Grad der Endgleichung in $y$ der Werth:

$$
\begin{gathered}
\mathrm{g}^{\prime}=\mu \beta_{0}+r_{1} h_{1}\left\{a_{t_{1}} \cdot \frac{1}{h_{1}}+m-t_{1}\right\}+r_{2} h_{2}\left\{a_{t_{2}} \cdot \frac{1}{h_{2}}+m-t_{2}\right\} \ldots . \\
+r_{\gamma} h_{\nu}\left\{a_{i_{\gamma}} \frac{1}{h_{\gamma}}+m-t_{\gamma}\right\}+m b_{0},
\end{gathered}
$$

welcher dem vorstehenden Werthe von $g$ gleich ist; w. z. b. w.

Man kann noch wünschen, in allen Fällen die Anzahl der endlichen Lösungen zu bestimmen, welche das System zulässt. Es ist auf besondere Relationen, welche zwischen den Zahlen-Coëfficienten der vorgelegten Gleichungen bestehen können, in gegenwärtigem Artikel bisher keine Rücksicht genommen; soll dies geschehen, so ist zu erwägen, dass es Systeme giebt, wie $f+\mu \varphi^{s}=0$ ( (wo $s$ eine ganze Zahl und $\mu$ ein numerischer Coëfficient ist), welche dem vorgelegten Systeme $f=0, \varphi=0$ gleichbedeutend sind, und dass man zwischen solchen eine Wahl treffen muss, um durch die Formel $\psi x=0$ die wahre Endgleichung in $x$ zu erhalten. Um Schwierigkeiten dieser Art zu vermeiden, denke man sich die Gleichungen $f=0, \varphi=0$ so beschaffen, dass es unmöglich sei, einen Exponenten $s$ und einen Coëfficienten $\mu$ (der auch eine ganze Function von $x$ oder $y$ sein kann) so zu bestimmen, dass, während die eine Gleichung $\varphi=0$ bleibt und die andere in $f+\mu^{s}{ }^{s}=0$ sich verwandelt, in 
Hinsicht auf die eine der Grössen $x$ oder $y$ von niedrigerem Grade sei als $f$, ohne zugleich in Hinsicht der andern den Grad von $f$ zu überschreiten. Mit andern Worten: wenn durch Hinzufügung von $\mu \varphi^{s}$ der Grad von $f$ nach $x$ (oder $y$ ) erniedrigt werden kann, ohne zugleich in Hinsicht auf $y$ (oder $x$ ) erhöht zu werden; so vollziehe man diese Reduction, bis ihre Fortsetzung unmöglich wird. Noch muss vorausgesetzt werden, dass die beiden Gleichungen keinen gemeinschaftlichen Factor haben; denn hätten sie einen, der z. B. $y$ enthielte, so bliebe $x$ offenbar unbestimmt, indem alsdann für jedes $x$ sich $y$ so bestimmen liesse, dass $f$ und $\varphi$ beide Null würden.

Es sei nun $g$ der Grad der Endgleichung in $x$, berechnet nach der im 22. Bande dieses Journals gegebenen Regel, mit Berücksichtigung der Zahlencoëfficienten in $f$ und $\varphi$, wie daselbst angedeutet ist; es sei ferner $g^{\prime \prime}$ der Grad der Endgleichung in $y$, die aus $f=0, \varphi=0$ hervorgeht, nach derselben Regel berechnet; endlich sei $g^{\prime \prime}$ der Grad der Endgleichung in $x$ des Systems $f+\delta y^{m}$ $+\varepsilon x^{\mu}=\mathbf{0}, \varphi=\mathbf{0}$, wo $\delta$ und $\varepsilon$ zwei unbestimmte Constanten sind und $\mu$ den Grad von $f$ nach $x$, so wie $m$ den nach $y$ bezeichnet (der Grad $g^{\prime \prime}$ muss ebenfalls nach der genannten Regel berechnet werden): so ist $g^{\prime \prime}$ auch der Grad der Endgleichung in $y$ des Systems $f+\delta y^{m}+\varepsilon x^{\mu}=0, \varphi=0$. Denn durch die Glieder $\delta y^{m}+\varepsilon x^{\mu}$ sind die gemeinschaftlichen Factoren der Coëfficienten der höchsten Potenzen von $x$ und $y$ in den nach $x$ und nach $y$ geordneten Gleichungen weggeschafft, und alsdann sind die Grade der Endgleichungen in $x$ und in $y$ gleich. Dieses vorausgesetzt, wird die Anzahl der endlichen Lösungen des Systems $f=0, \varphi=0$ durch $g+g^{\prime}-g^{\prime \prime}$ ausgedrückt. Denn das System $f+\delta y^{m}+\varepsilon x^{\mu}=0, \varphi=0$ hat $g^{\prime \prime}$ endliche Lösungen; wird aber $\delta=0$ und $\varepsilon=\mathbf{0}$ gesetzt, so werden $g^{\prime \prime}-g$ Werthe von $x$ und $g^{\prime \prime}-g^{\prime}$ Werthe von $y$ unendlich gross; und da es unmöglich ist, dass unendlich grosse Werthe von $x$ und $y$ zugleich dem Systeme $f=0, \varphi=0$ Genüge leisten, so sind $g^{\prime \prime}-g+g^{\prime \prime}-g^{\prime}$ Lösungen verloren gegangen und es bleiben nur $g+g^{\prime}-g^{\prime \prime}$, sämmtlich endliche Lösungen des Systems $f=0, \varphi=0$ übrig. 